\title{
Envelopes for Flight Through Stochastic Gusts
}

\author{
Johnhenri R. Richardson, ${ }^{*}$ Ella M. Atkins, ${ }^{\dagger}$ Pierre T. Kabamba ${ }^{\ddagger}$ and Anouck R. Girard ${ }^{\S}$ \\ University of Michigan, Ann Arbor, MI, 48109
}

We present a technique to guarantee, with a specified probability, that a steady flight state can be maintained when stochastic wind gusts act upon an airplane. First we linearize the airplane dynamic equations and present them as a linear time invariant system with the airplane's velocity and angular velocity as the state and gust velocity as the input. We then treat the gust velocity as a stationary random process and append the dynamics of a coloring filter to the linearized airplane equations so that the input is Gaussian white noise. We analyze the effect of the noise on the airplane's dynamics, using the resulting equations to quantify the effects of stochastic wind gusts on an airplane's steady flight envelope. During the analysis we introduce the notion of stationary flight and a stationary flight envelope to guarantee, within a specified probability, steady flight under such conditions. We also present a numerical example of a general aviation aircraft in steady level flight through moderate turbulence. In the example, the airplane's cruise speed fluctuates with a variance of $1.2 \mathrm{~m}^{2} / \mathrm{s}^{2}$ and we show examples of stationary flight envelopes for this case.

\section{Nomenclature}

$\mathbb{1}_{n} \quad$ Identity matrix of dimension $n$

A State matrix of Eq. (25)

$A_{\text {color }} \quad$ State matrix of the coloring filter

$A^{\mathrm{T}} \quad$ Transpose of some matrix $A$

$B \quad$ Input matrix of Eq. (25)

$B_{\text {color }} \quad$ Input matrix of the coloring filter

$C_{\text {color }}$ Output matrix of the coloring filter

$F_{a} \quad$ Sum of the forces acting on the airplane that depend on $v_{c}$ and $\omega$, i.e., aerodynamic forces

$F_{\text {other }} \quad$ Sum of the forces acting on the airplane that do not depend on $v_{c}$ and $\omega$

$h \quad$ Angular momentum of the airplane

$H_{\times} \quad$ Matrix formed from $h$ to replace a cross product with an inner product, see $\Omega_{\times}$

I Moment of inertia matrix of the airplane

$m \quad$ Mass of the airplane

$M_{a} \quad$ Sum of the moments acting on the airplane that depend on $v_{c}$ and $\omega$, i.e., aerodynamic moments

$M_{\text {other }} \quad$ Sum of the moments acting on the airplane that do not depend on $v_{c}$ and $\omega$

$n(t) \quad$ Gaussian white noise that drives the coloring filter

$\mathcal{N}\left(\mu, \sigma^{2}\right)$ Normal distribution with mean $\mu$ and variance $\sigma^{2}$

$P \quad$ Covariance matrix of the state vector in Eq. (25)

$P_{11} \quad(1,1)$ component of $P$, variance of $u$

$r \quad$ Position vector

$u \quad$ Longitudinal component of the airplane's velocity $v_{c}$

$v_{c} \quad$ Center of mass velocity of the airplane, $\left(\begin{array}{lll}u & v & w\end{array}\right)^{\mathrm{T}}$

$\dot{v}_{c} \quad$ Dotting a vector denotes a time derivative

$V_{c \times} \quad$ Matrix formed from $v_{c}$ to replace a cross product with an inner product, see $\Omega_{\times}$

*Ph.D. Candidate, Applied Physics Program, 450 Church St., Ann Arbor, MI, Student Member.

$\dagger$ Associate Professor, Department of Aerospace Engineering, 1320 Beal Ave, Ann Arbor, MI, Associate Fellow.

$\ddagger$ Professor, Department of Aerospace Engineering, 1320 Beal Ave, Ann Arbor, MI, Member.

$\S$ Assistant Professor, Department of Aerospace Engineering, 1320 Beal Ave, Ann Arbor, MI, Member. 


\begin{tabular}{|c|c|c|c|c|}
\hline$v_{w}$ & Gusts that perturb the steady state wind velocity & & & \\
\hline$\delta F_{a}$ & First order perturbation of $F_{a}$ after applying $v_{w}$ & & & \\
\hline$\delta M_{a}$ & First order perturbation of $M_{a}$ after applying $v_{w}$ & & & \\
\hline$\Delta v_{c}$ & Arbitrary perturbation of $v_{c}$ & & & \\
\hline$\delta v_{c}$ & First order perturbation of $v_{c}$ after applying $v_{w}$ & & & \\
\hline$\Delta \omega$ & Arbitrary perturbation of $\omega$ & & & \\
\hline$\delta \omega$ & First order perturbation of $\omega$ after applying $v_{w}$ & & & \\
\hline$\delta \Omega_{\times}$ & Matrix formed from $\delta \omega$ to replace a cross product with an inner product, see $\Omega_{\times}$ & & & \\
\hline$\rho(r)$ & Density of the airplane as a function of position & & & \\
\hline$\sigma_{n}^{2}$ & Variance of the noise input $n(t)$ & & & \\
\hline$\phi$ & State vector used in coloring filter & & & \\
\hline$\omega$ & Angular velocity of the airplane around its center of mass, $\left(\begin{array}{lll}p & q & r\end{array}\right)^{1}$ & & & \\
\hline \multirow{3}{*}{$\Omega_{\times}$} & \multirow{3}{*}{ Matrix formed from $\omega$ to replace a cross product with an inner product, $\Omega_{\times}(\omega)=$} & 0 & $-\omega_{3}$ & $\omega_{2}$ \\
\hline & & $\omega_{3}$ & 0 & $-\omega_{1}$ \\
\hline & & $-\omega_{2}$ & $\omega_{1}$ & 0 \\
\hline
\end{tabular}

\section{Introduction}

\section{A. Motivation}

In aviation, loss of control in-flight is a leading cause of accidents. In the years 2000-2009, 20 out of 89 commercial jet accidents, or $22 \%$, had loss of control in-flight identified as the primary cause. $^{2}$ Among 126 loss of control accidents that occurred between 1979 and 2009, investigations listed "stall/departure", meaning an excursion out of the flight envelope, as a causal or contributing factor in 49 cases, or $39 \%{ }^{3}$ The flight envelope, the set of speeds, altitudes, flight path angles, and bank angles at which an airplane can maintain steady flight, is a useful tool in identifying when an airplane is prone to loss of control. Steady flight, where the airplane's linear and angular velocity vectors are constant in the body frame, is not conducive to loss of control because most airplanes are designed to fly stably or stabilizably when flying steadily. Nevertheless, models of steady flight assume nominal conditions, conditions that cannot be expected in turbulence. To cope with this environmental uncertainty, pilots

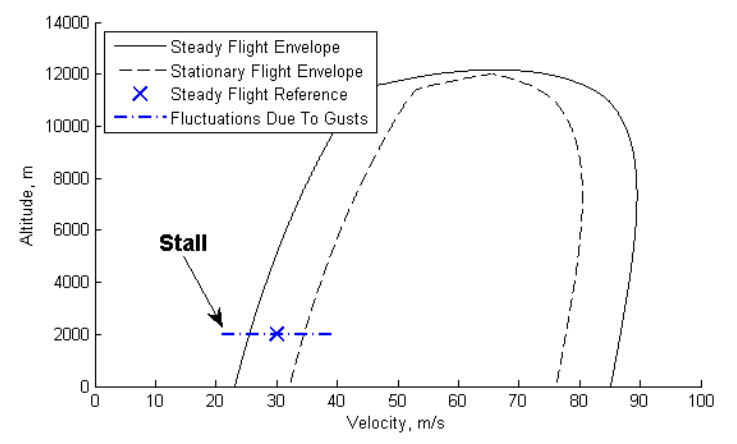
must fly more conservatively in turbulence than under nominal conditions, well within the steady flight envelope, or risk loss of control.

Consider the case of an airplane cruising close to its stall speed. If that airplane encountered turbulence, the forward component of its velocity would begin to fluctuate. Depending on turbulence intensity, the velocity could fluctuate sufficiently to cause the airplane to stall, as depicted in Figure 1. The figure shows a hypothetical case where turbulence causes fluctuations in airplane forward speed that have a standard deviation of $3 \mathrm{~m} / \mathrm{s}$. The dash-dot line shows three standard deviations worth of fluctuations around the steady flight state. Flying within the stationary flight envelope would ensure that the stall speed remained at least three standard deviations below the steady flight state, or that the plane's speed remained above the stall speed $99.9 \%$ of the time, assuming a Gaussian distribution.

The techniques described herein provide a tool to help clarify the boundaries of the flight envelope when faced with uncertain wind conditions. These techniques enable computation of the probability that a flight would stray beyond the boundaries of the steady flight envelope as a result of the uncertain wind conditions. In particular, we introduce the notions of stationary flight and stationary flight envelopes, the envelopes 
that result from adjustment of the steady flight envelope to take into account the effects of a stationary random process acting on the aircraft. Pilots can use the resulting tools to better understand their aircraft's capabilities and safe operating conditions in an uncertain environment.

\section{B. Prior Work and Present Contribution}

This paper primarily draws on two well-established fields: airplane dynamics and wind gust modeling. Detailed discussions of airplane dynamic equations and perturbations of these equations can be found in standard textbooks on the subject. ${ }^{4,5}$ Additional work has also been performed to model the effect of wind gusts $^{6,7}$ and wind shear 8,9 on aircraft dynamics. In this paper we take the traditional airplane dynamic equations and their linearization and express them in a format conducive to linear time invariant (LTI) system analysis with wind gusts as the input.

Several publications give detailed discussions of wind gust models and their power spectral densities, specifically the Dryden and von Kármán models. ${ }^{10,11}$ Discussions on spectral factorization of these wind models are also available. ${ }^{10}$ In this paper we apply these wind models to the aircraft dynamics and control problem described above.

By combining the work of the two fields we develop a tool to analyze how wind gusts affect steady flight. In particular, our results allow us to determine the probability that a particular maneuver remains safe despite stochastic gusts.

\section{Paper Organization}

We begin the paper by reviewing the standard airplane equations of motion and steady flight. We linearize the equations of motion around a steady flight condition with perturbations in the aerodynamic forces and moments as the input. We then show how the perturbations in aerodynamic forces and moments depend on stochastic wind gusts. This results in a LTI system with the airplane linear and angular velocities as the states, and the wind gusts as the input. Because the most widely accepted models of wind gusts are not white noise, we append to our LTI system a coloring filter that produces the wind gusts as its output from a stationary, Gaussian, white noise input. We then analyze the implications of the stochastic input on the airplane dynamics and give numerical examples for a general aviation aircraft. In the analysis and example we show what guarantees can be made about steady flight under uncertain wind conditions and introduce the notion of the stationary flight envelope.

\section{Developing the System of Equations}

\section{A. Linearizing the Airplane Equations of Motion}

We start with the airplane dynamic equations given by ${ }^{4}$

$$
\begin{aligned}
m\left(\dot{v}_{c}+\Omega_{\times} v_{c}\right) & =F_{a}\left(v_{c}, \omega\right)+F_{\text {other }}, \\
\dot{h}+\Omega_{\times} h & =M_{a}\left(v_{c}, \omega\right)+M_{\text {other }},
\end{aligned}
$$

where all the vectors are expressed in the body frame,

$$
h=I \omega,
$$

and

$$
I=\int_{\text {Volume }} \rho(r)\left(\|r\|^{2} \mathbb{1}_{3}-r r^{\mathrm{T}}\right) d V=\left(\begin{array}{ccc}
I_{x x} & -I_{x y} & -I_{x z} \\
-I_{x y} & I_{y y} & -I_{y z} \\
-I_{x z} & -I_{y z} & I_{z z}
\end{array}\right) .
$$

To understand $\Omega_{\times}$, note the following identity for the cross product of a pair of vectors expressed in an orthonormal coordinate system:

$$
\begin{gathered}
\boldsymbol{a} \times \boldsymbol{b}=A_{\times} b=B_{\times}^{\mathrm{T}} a \\
A_{\times}(a) \triangleq\left(\begin{array}{ccc}
0 & -a_{3} & a_{2} \\
a_{3} & 0 & -a_{1} \\
-a_{2} & a_{1} & 0
\end{array}\right)=-A_{\times}^{\mathrm{T}}(a) .
\end{gathered}
$$


The subscript $\times$ reminds us that this matrix replaced a cross product. $\Omega_{\times}$is therefore a matrix formed from the three components of $\omega$ as in Eq. (6). It is an alternative to the cross products commonly used in the airplane dynamic equations.

These airplane dynamic equations require a number of assumptions. They assume that the Earth is flat and that a point on the Earth's surface can be the origin of an inertial reference frame. They assume that the airplane's mass and moment of inertia do not change over time. They assume that the airplane is a rigid body and that it flies at a small angle of attack. They also assume still air, namely that air not yet disturbed by the airplane is fixed relative to the ground. Generalized equations exist that allow a nonzero but constant wind velocities.

In Eqs. (1) and (2) we have split the forces and moments into components that depend on velocity and angular velocity - typically the aerodynamic forces and moments - and components that do not. We perturb the airplane equations of motion to first order with the substitutions

$$
\begin{aligned}
F_{a}\left(v_{c}, \omega\right) & \rightarrow F_{a}\left(v_{c}+\delta v_{c}+v_{w}, \omega+\delta \omega\right) \approx F_{a}\left(v_{c}, \omega\right)+\delta F_{a}\left(\delta v_{c}, \delta \omega, v_{w}\right), \\
M_{a}\left(v_{c}, \omega\right) & \rightarrow M_{a}\left(v_{c}+\delta v_{c}+v_{w}, \omega+\delta \omega\right) \approx M_{a}\left(v_{c}, \omega\right)+\delta M_{a}\left(\delta v_{c}, \delta \omega, v_{w}\right) .
\end{aligned}
$$

We could also include an input $\omega_{w}$, the gusts' angular velocity, but here opt to consider only the gusts' linear velocity. Reference 11 gives criteria based on airplane stability derivatives to judge when the angular velocity components should be included. Because of the wind velocity perturbations' impact on the velocity and angular velocity, we also need to make the substitutions

$$
v_{c} \rightarrow v_{c}+\delta v_{c}, \quad \omega \rightarrow \omega+\delta \omega .
$$

The angular velocity substitution will also require a perturbation of $\Omega_{\times}$to $\tilde{\Omega}_{\times}(\omega+\delta \omega)$. The reader can verify using Eq. (6) that

$$
\tilde{\Omega}_{\times}(\omega+\delta \omega)=\Omega_{\times}(\omega)+\delta \Omega_{\times}(\delta \omega) .
$$

After substituting all of these perturbations into Eqs. (1) and (2), cancelling the steady state terms, and ignoring second order terms, we get

$$
\begin{aligned}
\delta F_{a} & \approx m\left(\delta \dot{v}_{c}+\delta \Omega_{\times} v_{c}+\Omega_{\times} \delta v_{c}\right), \\
\delta M_{a} & \approx I \delta \dot{\omega}+\delta \Omega_{\times} h+\Omega_{\times} I \delta \omega .
\end{aligned}
$$

Note that in Eq. (5) when we convert a cross product into an inner product, either vector from the cross product can be converted into a matrix. We therefore define $V_{c \times}$ and $H_{\times}$in the same manner as $\Omega_{\times}$and replace $\delta \Omega_{\times} v_{c}$ and $\delta \Omega_{\times} h$ with $-V_{c \times} \delta \omega$ and $-H_{\times} \delta \omega$, respectively. After making these substitutions and rearranging terms, the linearized equations become

$$
\begin{aligned}
\delta \dot{v}_{c} & =V_{c \times} \delta \omega-\Omega_{\times} \delta v_{c}+\frac{1}{m} \delta F_{a}, \\
I \delta \dot{\omega} & =H_{\times} \delta \omega-\Omega_{\times} I \delta \omega+\delta M_{a} .
\end{aligned}
$$

We can realistically assume that the airplane's moment of inertia is positive definite and therefore invertible and can be moved to the right hand side of Eq. (14). Finally, we combine the two linearized equations, resulting in the LTI system

$$
\left(\begin{array}{c}
\delta \dot{v}_{c} \\
\delta \dot{\omega}
\end{array}\right)=\left(\begin{array}{cc}
-\Omega_{\times} & V_{c \times} \\
0 & I^{-1}\left(H_{\times}-\Omega_{\times} I\right)
\end{array}\right)\left(\begin{array}{c}
\delta v_{c} \\
\delta \omega
\end{array}\right)+\left(\begin{array}{cc}
\frac{1}{m} \mathbb{1}_{3} & 0 \\
0 & I^{-1}
\end{array}\right)\left(\begin{array}{c}
\delta F_{a} \\
\delta M_{a}
\end{array}\right),
$$

with the force and moment perturbations as the input and the airplane's linear and angular velocity perturbations as the state. The only assumption we relax in the linearization is the assumption that the wind velocity is constant, now allowing the small $v_{w}$ to perturb it. We still ignore any structural modes that the gusts could excite.

\section{B. Perturbing the Forces \& Moments}

Next we determine how to relate $\delta F_{a}$ and $\delta M_{a}$ to $v_{w}$. Consider an arbitrary perturbation to $v_{c}$ and $\omega$ where $v_{c} \rightarrow v_{c}+\Delta v_{c}$ and $\omega \rightarrow \omega+\Delta \omega$. For small perturbations $F_{a}$ responds as

$$
F_{a}\left(v_{c}+\Delta v_{c}, \omega+\Delta \omega\right) \approx F_{a}\left(v_{c}\right)+\delta F_{a}\left(\Delta v_{c}, \Delta \omega\right),
$$


where, to first order, the perturbations to the force and similarly to the moment are

$$
\begin{aligned}
\delta F_{a}\left(\Delta v_{c}, \Delta \omega\right) & =\frac{\partial F_{a}}{\partial v_{c}} \Delta v_{c}+\frac{\partial F_{a}}{\partial \omega} \Delta \omega, \\
\delta M_{a}\left(\Delta v_{c}, \Delta \omega\right) & =\frac{\partial M_{a}}{\partial v_{c}} \Delta v_{c}+\frac{\partial M_{a}}{\partial \omega} \Delta \omega .
\end{aligned}
$$

The partial derivatives above are evaluated at the reference condition and consist of airplane stability derivatives. We can consider $\delta v_{c}$ and $v_{w}$ to each be independent perturbations of $v_{c}$ akin to $\Delta v_{c}$ above, and $\delta \omega$ to be a perturbation to $\omega$ akin to $\Delta \omega$. As a result we get

$$
\begin{aligned}
\delta F_{a}\left(\delta v_{c}, \delta \omega, v_{w}\right) & =\frac{\partial F_{a}}{\partial v_{c}}\left(\delta v_{c}+v_{w}\right)+\frac{\partial F_{a}}{\partial \omega} \delta \omega, \\
\delta M_{a}\left(\delta v_{c}, \delta \omega, v_{w}\right) & =\frac{\partial M_{a}}{\partial v_{c}}\left(\delta v_{c}+v_{w}\right)+\frac{\partial M_{a}}{\partial \omega} \delta \omega .
\end{aligned}
$$

Making these substitutions, Eq. (15) becomes

$$
\left(\begin{array}{c}
\delta \dot{v}_{c} \\
\delta \dot{\omega}
\end{array}\right)=\left(\begin{array}{cc}
-\Omega_{\times}+\frac{1}{m} \frac{\partial F_{a}}{\partial v_{c}} & V_{c \times}+\frac{1}{m} \frac{\partial F_{a}}{\partial \omega} \\
I^{-1} \frac{\partial M_{a}}{\partial v_{c}} & I^{-1}\left(H_{\times}-\Omega_{\times} I+\frac{\partial M_{a}}{\partial \omega}\right)
\end{array}\right)\left(\begin{array}{c}
\delta v_{c} \\
\delta \omega
\end{array}\right)+\left(\begin{array}{c}
\frac{1}{m} \frac{\partial F_{a}}{\partial v_{c}} \\
I^{-1} \frac{\partial M_{a}}{\partial v_{c}}
\end{array}\right) v_{w}
$$

a LTI system with the velocity of the gusts as the input. Note that Eq. (21) does not assume that $v_{w}$ is a random process. We can also use these equations to study the impulse response of an airplane to changes in wind velocity or to study the step responses to wind shear events.

Note that we have only perturbed the force and moment with respect to velocity and angular velocity. We are, as a result, assuming that the force and moment do not depend on the linear or angular acceleration. We also assume that the system is stable with the inclusion of those partial derivatives in the state matrix. Note as well that we do not include any control inputs; the inputs are assumed to remain at the values corresponding to the reference condition. For the stability derivatives, details on how to compute them are available in Chapter 3 of Ref. 4.

\section{Modeling the Gusts}

Until this point the only assumption we have made about $v_{w}$ is that it is small. This was necessary to discard higher order terms in the linearization. For our application, we model uncertainty in the airplane's velocity by substituting for $v_{w}$ a stationary random process. Such a definition of gusts is generally consistent with turbulence found in clear air or storms but is not appropriate for modeling wind shear or discrete gusts. Engineers typically use one of two models of stochastic wind gusts: the Dryden and von Kármán models. ${ }^{10,11}$ Both models define the random gusts in terms of their power spectral densities and in both cases the random process defined by the power spectral density is colored. For the purposes of our analysis we would prefer to work with white noise. Let us define the spectral density of $v_{w}$ to be $\Phi_{w}$ and assume that it has a rational spectral factorization. This assumption is valid for the Dryden model but can only be approximated for the von Kármán model. ${ }^{10}$ Once spectrally factorized, $v_{w}$ is given as the output of a coloring filter driven by Gaussian white noise $n(t)$ with zero mean and variance $\sigma_{n}^{2}$. We write this filter as

$$
\begin{aligned}
\dot{\phi} & =A_{\text {color }} \phi+B_{\text {color }} n(t), \\
v_{w} & =C_{\text {color }} \phi, \\
n(t) & =\mathcal{N}\left(0, \sigma_{n}^{2}\right) .
\end{aligned}
$$

Such a filter can be derived from the power spectral densities of the Dryden or von Kármán models given in Ref. 11.

\section{Combining the Models}

We can append the filter from the previous section to Eq. (21) and get the system of equations

$$
\left(\begin{array}{c}
\delta \dot{v}_{c} \\
\delta \dot{\omega} \\
\dot{\phi}
\end{array}\right)=\left(\begin{array}{ccc}
-\Omega_{\times}+\frac{1}{m} \frac{\partial F_{a}}{\partial v_{c}} & V_{c \times}+\frac{1}{m} \frac{\partial F_{a}}{\partial \omega} & \frac{1}{m} \frac{\partial F_{a}}{\partial v_{c}} C_{c o l o r} \\
I^{-1} \frac{\partial M_{a}}{\partial v_{c}} & I^{-1}\left(H_{\times}-\Omega_{\times} I+\frac{\partial M_{a}}{\partial \omega}\right) & I^{-1} \frac{\partial M_{a}}{\partial v_{c}} C_{c o l o r} \\
0 & 0 & A_{\text {color }}
\end{array}\right)\left(\begin{array}{c}
\delta v_{c} \\
\delta \omega \\
\phi
\end{array}\right)+\left(\begin{array}{c}
0 \\
0 \\
B_{\text {color }}
\end{array}\right) n(t),
$$


or more compactly,

$$
\left(\begin{array}{c}
\delta \dot{v}_{c} \\
\delta \dot{\omega} \\
\dot{\phi}
\end{array}\right)=A\left(\begin{array}{c}
\delta v_{c} \\
\delta \omega \\
\phi
\end{array}\right)+B n(t) .
$$

In most applications the state matrix $A$ is asymptotically stable because the state matrices of Eq. (21) and the coloring filter are asymptotically stable. Since the system given by Eq. (25) is linear, asymptotically stable, and driven by zero-mean, Gaussian white noise, the perturbations $\delta v_{c}$ and $\delta \omega$ are also zero-mean, Gaussian, stationary random processes. For a white noise input to Eq. (25), the steady state covariance $P$ of this system's state is finite and given by the solution of the Lyapunov equation

$$
A P+P A^{\mathrm{T}}+B \sigma_{n}^{2} B^{\mathrm{T}}=0 .
$$

The diagonal terms of $P$ are of particular interest as they represent the variances of the state variables. Given the dynamics of the noise filter and the variance of the white noise that drives it, we can determine the variances of the components of the linear and angular velocities. This allows us to determine the probability that the airplane strays outside the flight envelope when subject to turbulence.

\section{Stationary Flight and the Stationary Flight Envelope}

This section describes an application of the model just derived. The application centers on a Navion general aviation aircraft model from Ref. 4. The necessary parameters are summarized in Table 1 in the appendix. To calculate the flight envelopes, we use the algorithm published in Chapter 14 of Ref. 1.

\section{A. Utility of the Model}

Once we know the covariance matrix of the system defined by Eq. (25) we can quantify the effect of wind gusts on steady flight. Consider the cruise example discussed earlier. For steady level flight the reference condition is

$$
v_{c}=\left(\begin{array}{l}
u \\
0 \\
0
\end{array}\right), \quad \omega=\left(\begin{array}{l}
0 \\
0 \\
0
\end{array}\right) .
$$

The top left term $P_{11}$ of the covariance matrix $P$ is the variance of the airplane's cruise speed $u$. If the airplane were cruising at a point in its flight envelope close to stall and encountered turbulence, then $P_{11}$ could be used to compute the probability of stalling. The angle of attack and sideslip angle will also fluctuate, but here we assume stall will be driven by changes in $u$. If we call the longitudinal component of $\delta v_{c} \delta u$ then we can say that $u+\delta u$ is a random process with the Gaussian distribution $\mathcal{N}\left(u, P_{11}\right)$. The probability p that at a particular instant the process' value is less than $N$ standard deviations below the mean can be computed from the Gaussian distribution's cumulative distribution function and is given by

$$
\mathrm{p}\left(u+\delta u \leq u-N \sqrt{P_{11}}\right)=\frac{1}{2}\left(1-\operatorname{erf}\left(\frac{N}{\sqrt{2}}\right)\right),
$$

where $\operatorname{erf}\left(\frac{N}{\sqrt{2}}\right)$ is the error function. So, for example, if the stall speed were exactly two standard deviations less than $u$, i.e., $N=2$, then as the cruise speed randomly fluctuated the airplane would slow to below the stall speed at most $2.3 \%$ of the time. If it were three standard deviations less than $u$, the plane would slow below the stall speed $0.13 \%$ of the time. Figure 2 gives an illustration. We could also consider other excursions from the flight envelope, such as exceeding the airplane's thrust limit on the right side of the envelope, but for now limit our discussion to stall because it has greater implications on loss of control.

To help predict safe flight conditions when facing turbulence we propose the definition and use of the terms stationary flight and stationary flight envelope. Stationary flight is a flight state where the airplane's velocity and angular velocity are stationary random processes. Equation (25) is itself an example of a system whose flight state is stationary. A stationary flight envelope is an adjustment of the steady flight envelope for use in the case of stationary flight. It involves shifting the boundaries of the steady flight envelope as a function of the variances of the airplane's linear and angular velocities to achieve a desired safety margin. For the case of cruise we can form a stationary flight envelope by shifting the steady flight envelope inward 


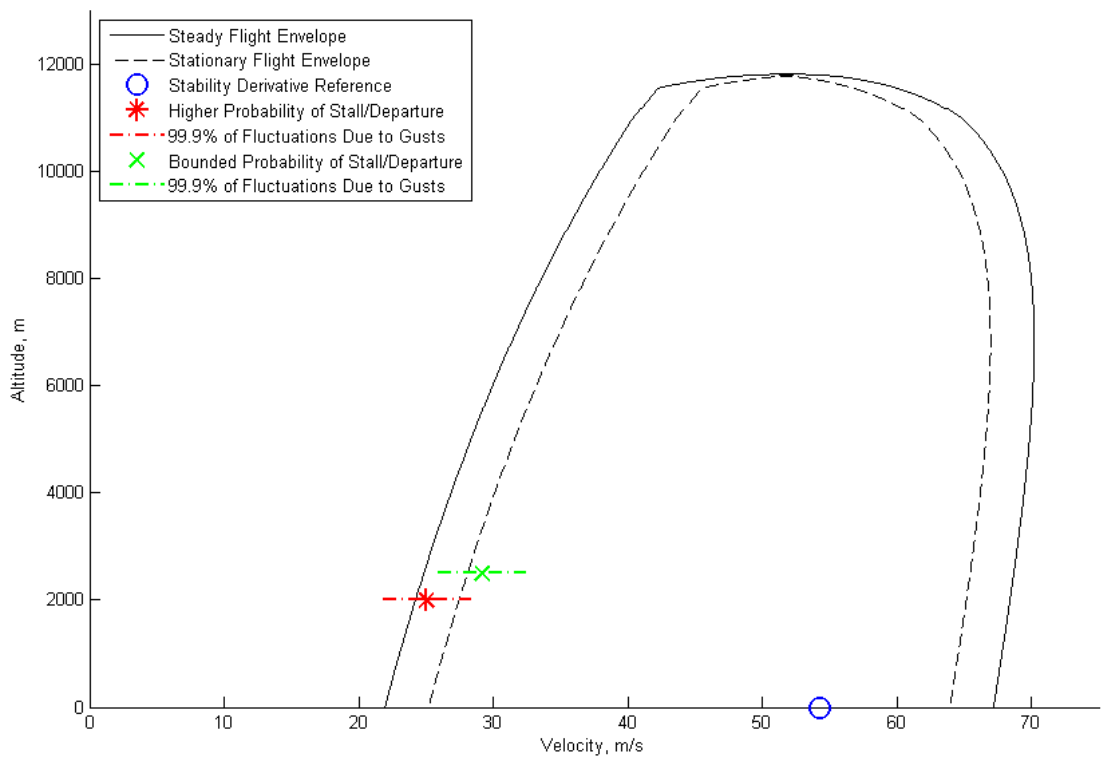

Figure 2. Steady and stationary flight envelopes for a general aviation aircraft in moderate turbulence where the stationary flight envelope is shifted inward by $3 \sqrt{P_{11}}$. Also shown are two reference steady flight states. For the state inside the stationary flight envelope, as the cruise speed fluctuates due to the turbulence, at most $\mathbf{0 . 1 3 \%}$ of the time the cruise speed strays outside the steady flight envelope. The state outside the stationary flight envelope spends between $0.13 \%$ and $50 \%$ of the time with its cruise speed outside of the steady flight envelope.

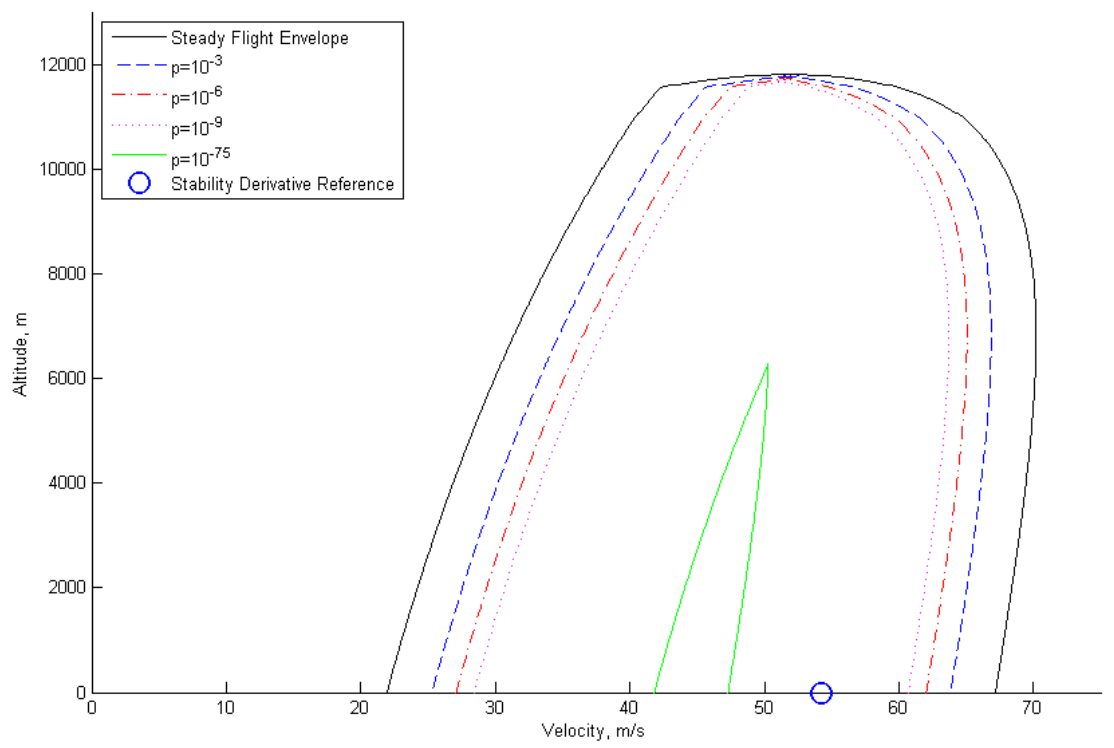

Figure 3. Steady flight envelope for a general aviation aircraft and several stationary flight envelopes. For each stationary flight envelope the turbulence is the same but the safety margin, the percentage of fluctuations extending outside of the steady flight envelope, changes. 
by three standard deviations of the fluctuations of the cruise speed, as shown in Figure 2. When planning a flight through known turbulent conditions or upon encountering turbulence and flight state perturbations whose statistics can be estimated, a pilot can adjust the airplane's speed and altitude to ensure that the flight lies within the stationary flight envelope. For an inward shift of three standard deviations, flying within the stationary flight envelope would guarantee that the airplane would stay within the steady flight envelope at least $99.9 \%$ of the time. Of course our choice of a three standard deviation adjustment is arbitrary and can be changed to generate the desired safety margin.

\section{B. Numerical Example: General Aviation Aircraft}

We now give a numerical example using the Navion general aviation aircraft model, detailed in Table 1 in the appendix. We consider the case of steady level cruise in moderate turbulence. We use high altitude turbulence, which Ref. 11 defines as valid at 2,000 ft above terrain. For the noise we use a filter for continuous Dryden gusts given in Ref. 12. The parameters chosen for the airplane's flight state and the coloring filter are given in Table 2, also in the appendix. Given this airplane model, flight state, and turbulence model, we compute the variance of the airplane's forward speed, $P_{11}$, to be $1.2 \mathrm{~m}^{2} / \mathrm{s}^{2}$. With this information we can plot the stationary flight envelope by shifting the steady flight envelope inwards by three standard deviations, $3 \sqrt{P_{11}}$, as shown in Figure 2. For computational simplicity we assume that $P_{11}$ remains constant throughout the envelope. This assumption appears to be valid because varying the reference cruise speed across the entire envelope only changes $P_{11}$ by $0.1 \%$. However, our sources do not quantify how the stability derivatives and other airplane parameters vary throughout the envelope.

For other choices of safety margin, the stationary flight envelope is a different size. Equation (29) shows the relationship between probability of departing the steady flight envelope and how many standard deviations below $u$ the stall speed is. Figure 3 shows several stationary flight envelopes corresponding to different probabilities of instantaneously dropping below the stall speed. Note that as you consider tighter and tighter safety margins, the probability of departing the envelope decreases faster than the number of standard deviations. Decreasing the probability by an additional factor of $10^{-3}$ results in less than three standard deviations worth of additional inward shift to draw the resulting stationary flight envelope. Choosing a probability that is sufficiently small does eventually reduce the flight envelope dramatically. Also note that the steady flight envelope is itself a stationary flight envelope with a $50 \%$ probability of instantaneously departing the steady flight envelope as the cruise speed fluctuates.

This example demonstrates how the steady flight envelope can be adjusted based on known turbulent conditions and a specified safety margin. We expect that for an airplane model with a smaller steady flight envelope, like a light unmanned aerial vehicle, the reduction of the flight envelope would be even more pronounced.

\section{Conclusion}

As we have shown, by using linearized airplane dynamic equations and driving them with random wind gusts we can estimate the probability of violating the steady flight constraints. This information can ultimately be used, given wind gust statistics, to choose flight states that guarantee, with specified probability, that the aircraft remains within the steady flight envelope despite uncertain conditions. In particular, we propose shifting the boundary of the steady flight envelope based on the statistics of the wind gusts to provide a well-defined safety margin when flying through turbulence. We argue that the stationary flight envelope can help pilots or autopilots plan safe flights through known turbulent conditions. Similarly, if airplanes encounter turbulent conditions in-flight and can estimate the statistics of the turbulence or the flight state, the stationary flight envelope can give pilots a basis to determine quantitatively which maneuvers are safe.

We see this framework as a promising route toward advancing the notion of steady flight into situations where it did not previously apply. In the future we will continue to extend the notion of the steady flight envelope in uncertain environments. For instance, we can consider use of the stationary flight envelope to predict the probability of loss of control in other phases of flight besides cruise, to design closed-loop control systems robust to turbulence, and to analyze a different departure from the flight envelope, namely excessive loading of an airplane's structure. 


\section{Appendix: Parameters for the Numerical Example}

In our numerical example we use a Navion general aviation aircraft model from Ref. 4. Table 1 summarizes the necessary airplane parameters. The parameters provided by this source correspond to a steady flight state where $u=54.2 \mathrm{~m} / \mathrm{s}$ and the altitude is mean sea level. For parameters not specified in Ref. 4, we use the value given in Ref. 1's general aviation aircraft model. The last five parameters in the table are not given in either source. We based the last two on a plot for a different airplane given in Ref. 5 . The remaining three parameters did not seem to have an impact on the results of this example and we set them as zero. Table 2 summarizes the flight state and turbulence parameters used in our example.

Table 1. General aviation aircraft parameters and stability derivatives needed in our analysis.

Values are given in the units used by the source.

\begin{tabular}{|c|c|c|c|c|c|c|}
\hline Symbol & Value & Name (Where Applicable) & Ref. & Symbol & Value & Ref. \\
\hline$W$ & $2,750 \mathrm{lbs}$ & Weight & 4 & $C_{L_{\alpha}}$ & 4.44 & 4 \\
\hline$I_{x x}$ & 1,048 slug $\mathrm{ft}^{2}$ & Rolling Moment of Inertia & 4 & $C_{D_{\alpha}}$ & 0.33 & 4 \\
\hline$I_{y y}$ & 3,000 slug $\mathrm{ft}^{2}$ & Pitching Moment of Inertia & 4 & $C_{m_{\alpha}}$ & -0.683 & 4 \\
\hline$I_{z z}$ & 3,530 slug $\mathrm{ft}^{2}$ & Yawing Moment of Inertia & 4 & $C_{m_{q}}$ & -9.96 & 4 \\
\hline$I_{x z}$ & 0 slug $\mathrm{ft}^{2}$ & Product of Inertia About $x z$ Axis & 4 & $C_{y_{\beta}}$ & -0.564 & 4 \\
\hline$I_{x y}, I_{y z}$ & 0 & Other Products of Inertia & 4 & $C_{l_{\beta}}$ & -0.074 & 4 \\
\hline$S$ & $184 \mathrm{ft}^{2}$ & Wing Planform Area & 4 & $C_{n_{\beta}}$ & $0.071^{*}$ & 4 \\
\hline$b$ & $33.4 \mathrm{ft}$ & Wing Span & 4 & $C_{l_{p}}$ & -0.410 & 4 \\
\hline $\bar{c}$ & $5.7 \mathrm{ft}$ & Mean Chord & 4 & $C_{n_{p}}$ & 0.0575 & 4 \\
\hline$e$ & 0.8 & Oswald Efficiency Factor & 1 & $C_{l_{r}}$ & 0.107 & 4 \\
\hline$a$ & 0.6 & Air Density Exponent & 1 & $C_{n_{r}}$ & -0.125 & 4 \\
\hline$P_{\max }^{s}$ & $290 \mathrm{hp}$ & Maximum Engine Power & 1 & $C_{y_{p}}$ & 0 & \\
\hline$C_{L_{\max }}$ & 2.4 & Maximum Coefficient of Lift & 1 & $C_{y_{r}}$ & 0 & \\
\hline$\eta$ & 0.8 & Propeller Efficiency & 1 & $C_{z_{q}}$ & 0 & \\
\hline$C_{L}$ & 0.41 & Coefficient of Lift & 4 & $\frac{d C_{D}^{2}}{d M}{ }^{\dagger}$ & 0 & 5 \\
\hline$C_{D}$ & .05 & Coefficient of Drag & 4 & $\frac{d C_{m}}{d M} \dagger$ & 0 & 5 \\
\hline
\end{tabular}

${ }^{*}$ The first and second editions of Ref. 4 disagree on the sign of $C_{n_{\beta}}$ for this airplane model. We have chosen the positive sign from the first edition, which gives a stable state matrix.

${ }^{\dagger} \mathrm{M}$ denotes the Mach number.

Table 2. Parameters describing the flight state and moderate turbulence. Values are given in units used by the source.

\begin{tabular}{lclc} 
Symbol & Value & Name (Where Applicable) & Ref. \\
\hline$u$ & $54.2 \mathrm{~m} / \mathrm{s}$ & Airplane Cruise Speed & N/A \\
$\sigma_{n}^{2}$ & 1 & Noise Variance & 12 \\
$\sigma_{u}$ & $20 \mathrm{ft} / \mathrm{s}$ & Longitudinal Turbulence Intensity & 11 \\
$\sigma_{v}, \sigma_{w}$ & $\sigma_{u}$ & Other Turbulence Intensities & 11 \\
$L_{u}$ & $1,750 \mathrm{ft}$ & Longitudinal Turbulence Scale Length & 11 \\
$L_{v}, L_{w}$ & $\frac{L_{u}}{2}$ & Other Turbulence Scale Lengths & 11
\end{tabular}

\section{Acknowledgments}

This work was supported in part by the Department of Defense under Air Force grant number FA 8650-07-2-3744 and through the National Defense Science \& Engineering Graduate Fellowship Program. 


\section{References}

${ }^{1}$ McClamroch, N. H., Steady Aircraft Flight and Performance, Princeton University Press, Princeton, 2011. 2010.

${ }^{2}$ Aviation Safety, "Statistical Summary of Commercial Jet Airplane Accidents," Tech. rep., Boeing Commercial Airplanes,

${ }^{3}$ Belcastro, C. M. and Foster, J. V., "Aircraft Loss-of-Control Accident Analysis," AIAA Guidance, Navigation, and Control Conference, AIAA, Toronto, ON, 2010.

${ }^{4}$ Nelson, R. C., Flight Stability and Automatic Control, WCB/McGraw-Hill, Boston, 2nd ed., 1998.

${ }^{5}$ Roskam, J., Airplane Flight Dynamics and Automatic Flight Controls, Vol. 1, Design, Analysis, and Research Corporation, Lawrence, KS, 2003.

${ }^{6}$ Etkin, B., "Turbulent Wind and Its Effect on Flight," J. of Aicraft, Vol. 18, No. 5, 1981, pp. 327-345.

${ }^{7}$ Davison, D., Kabamba, P., and Meerkov, S., "Robustness with Respect to Disturbance Model Uncertainty: Theory and Application to Autopilot Performance Analysis," Mathematical Problems in Engineering, Vol. 6, 2000, pp. 267-304.

${ }^{8}$ Mulgund, S. and Stengel, R., "Optimal Recovery from Microburst Wind Shear," Journal of Guidance, Control, and Dynamics, Vol. 16, No. 6, 1993, pp. 1010-1017.

${ }^{9}$ Hinton, D. A., "Forward-Look Wind-Shear Detection for Microburst Recovery," Journal of Aircraft, Vol. 29, No. 1, 1992, pp. 63-66.

${ }^{10}$ Hoblit, F. M., Gust Loads on Aircraft: Concepts and Applications, AIAA Education Series, Washington, DC, 1988.

${ }^{11}$ MIL-HDBK-1797, Flying Qualities of Piloted Aircraft, U.S. Dept. of Defense, 1997.

12 "Dryden Wind Turbulence Model (Continuous)", MATLAB Reference Pages, The MathWorks, Inc., 2010. 